\title{
Topological Black Holes in Brans-Dicke-Maxwell Theory
}

\author{
A. Sheykhi1 ${ }^{1,2 *}$ and H. Alavirad ${ }^{1}$ \\ ${ }^{1}$ Department of Physics, Shahid Bahonar University, P.O. Box 76175, Kerman, Iran \\ ${ }^{2}$ Research Institute for Astronomy and Astrophysics of Maragha (RIAAM), Maragha, Iran
}

We derive a new analytic solution of $(n+1)$-dimensional $(n \geq 4)$ Brans-DikceMaxwell theory in the presence of a potential for the scalar field, by applying a conformal transformation to the dilaton gravity theory. These solutions describe topological charged black holes with unusual asymptotics. We obtain the conserved and thermodynamic quantities through the use of the Euclidean action method. We also study the thermodynamics of the solutions and verify that the conserved and thermodynamic quantities of the solutions satisfy the first law of black hole thermodynamics.

Keywords: Brans-Dicke; dilaton; black holes.

\section{INTRODUCTION}

Among the alternative theories of general relativity, perhaps, the most well-known theory is the scalar-tensor theory which was pioneered several decades ago by Brans and Dicke [1], who sought to incorporate Mach's principle into gravity. Compared to Einstein's general relativity, Brans-Dicke (BD) theory describes the gravitation in terms of the metric as well as scalar fields and accommodates both Mach's principle and Dirac's large number hypothesis as new ingredients. Although BD theory has passed all the possible observational tests [2], however, the singularity problem remains yet in this theory. In recent years this theory got a new impetus as it arises naturally as the low energy limit of many theories of quantum gravity such as the supersymmetric string theory or the Kaluza-Klein theory. Besides, recent observations show that at the present epoch, our Universe expands with acceleration instead of deceleration along the scheme of standard Friedmann models and since general relativity could not describe such Universe correctly, cosmologists have attended to alternative theories of gravity such as BD theory. Due to highly nonlinear character of BD theory, a desirable pre-

\footnotetext{
*sheykhi@mail.uk.ac.ir
} 
requisite for studying strong field situation is to have knowledge of exact explicit solutions of the field equations. And as black holes are very important both in classical and quantum gravity, many authors have investigated various aspects of them in BD theory [3, 4, 5]. It turned out that the dynamic scalar field in the BD theory plays an important role in the process of collapse and critical phenomenon. The first four-dimensional black hole solutions of BD theory was obtained by Brans in four classes [6]. It has been shown that among these four classes of the static spherically symmetric solutions of the vacuum BD theory of gravity only two are really independent, and only one of them is permitted for all values of $\omega$. It has been proved that in four dimensions, the stationary and vacuum BD solution is just the Kerr solution with constant scalar field everywhere [7]. It has been shown that the charged black hole solution in four-dimensional Brans-Dicke-Maxwell (BDM) theory is just the Reissner-Nordstrom solution with a constant scalar field, however, in higher dimensions, one obtains the black hole solutions with a nontrivial scalar field [8]. This is because the stress energy tensor of Maxwell field is not traceless in the higher dimensions and the action of Maxwell field is not invariant under conformal transformations. Accordingly, the Maxwell field can be regarded as the source of the scalar field in the BD theory [8]. Other studies on black hole solutions in BD theory have been carried out in [9, 10, 11, 12].

On another front, it has been realized that in four dimensions the topology of the event horizon of an asymptotically flat stationary black hole is uniquely determined to be the two-sphere $S^{2}$ [13, 14]. The "topological censorship theorem" of Friedmann, Schleich and Witt indicates the impossibility of non spherical horizons [15, 16]. However, when the asymptotic flatness of spacetime is violated, there is no fundamental reason to forbid the existence of static or stationary black holes with nontrivial topologies. It was shown that for asymptotically AdS spacetime, in the four-dimensional Einstein-Maxwell theory, there exist black hole solutions whose event horizons may have zero or negative constant curvature and their topologies are no longer the two-sphere $S^{2}$. The properties of these black holes are quite different from those of black holes with usual spherical topology horizon, due to the different topological structures of the event horizons. Besides, the black hole thermodynamics is drastically affected by the topology of the event horizon. It was argued that the HawkingPage phase transition [17] for the Schwarzschild-AdS black hole does not occur for locally AdS black holes whose horizons have vanishing or negative constant curvature, and they are thermally stable [18]. The studies on the topological black holes have been carried out 
extensively in many aspects $19,20,21,22,23,24,25,26,27,28,29,30,31,32,33,34$, 35, 36, 37]. In this paper, we would like to study the topological black hole solutions in $(n+1)$-dimensional BDM theory for an arbitrary value of $\omega$ and investigate their properties.

In the next section, we review the basic equations and the conformal transformation between the action of the dilaton gravity theory and the BD theory. In section III, we construct charged topological black hole solutions in BDM theory and investigate their properties. In section IV, we study the thermodynamical properties of the solutions and calculate the conserved quantities through the use of the Euclidean action method. The last section is devoted to summary and discussion.

\section{BASIC EQUATIONS AND CONFORMAL TRANSFORMATIONS}

The action of the $(n+1)$-dimensional Brans-Dicke-Maxwell theory with one scalar field $\Phi$ and a self-interacting potential $V(\Phi)$ can be written as

$$
I_{G}=-\frac{1}{16 \pi} \int_{\mathcal{M}} d^{n+1} x \sqrt{-g}\left(\Phi R-\frac{\omega}{\Phi}(\nabla \Phi)^{2}-V(\Phi)-F_{\mu \nu} F^{\mu \nu}\right)-\frac{1}{8 \pi} \int d^{n} x \sqrt{h} \Phi K,
$$

where $R$ is the scalar curvature, $V(\Phi)$ is a potential for the scalar field $\Phi, F_{\mu \nu}=\partial_{\mu} A_{\nu}-\partial_{\nu} A_{\mu}$ is the electromagnetic field tensor, and $A_{\mu}$ is the electromagnetic potential. The factor $\omega$ is the coupling constant. The last term in Eq. (11) is the Gibbons-Hawking surface term. It is required for the variational principle to be well-defined. The factor $K$ represents the trace of the extrinsic curvature for the boundary and $h$ is the induced metric on the boundary. The equations of motion can be obtained by varying the action (1) with respect to the gravitational field $g_{\mu \nu}$, the scalar field $\Phi$ and the gauge field $A_{\mu}$ which yields the following field equations

$$
\begin{aligned}
& G_{\mu \nu}=\frac{\omega}{\Phi^{2}}\left(\nabla_{\mu} \Phi \nabla_{\nu} \Phi-\frac{1}{2} g_{\mu \nu}(\nabla \Phi)^{2}\right)-\frac{V(\Phi)}{2 \Phi} g_{\mu \nu}+\frac{1}{\Phi}\left(\nabla_{\mu} \nabla_{\nu} \Phi-g_{\mu \nu} \nabla^{2} \Phi\right) \\
& +\frac{2}{\Phi}\left(F_{\mu \lambda} F_{\nu}{ }^{\lambda}-\frac{1}{4} F_{\rho \sigma} F^{\rho \sigma} g_{\mu \nu}\right), \\
& \nabla^{2} \Phi=-\frac{n-3}{2(n-1) \omega+2 n} F^{2}+\frac{1}{2(n-1) \omega+2 n}\left((n-1) \Phi \frac{d V(\Phi)}{d \Phi}-(n+1) V(\Phi)\right), \\
& \nabla_{\mu} F^{\mu \nu}=0
\end{aligned}
$$

where $G_{\mu \nu}$ and $\nabla$ are, respectively, the Einstein tensor and covariant differentiation in the spacetime metric $g_{\mu \nu}$. It is apparent that the right hand side of Eq. (22) includes the second 
derivatives of the scalar field, so it is hard to solve the field equations (2)-(44) directly. We can remove this difficulty by a conformal transformation. Indeed, the BDM theory (11) can be transformed into the Einstein-Maxwell theory with a minimally coupled scalar field via the conformal transformation

$$
\begin{aligned}
& \bar{g}_{\mu \nu}=\Phi^{\frac{2}{n-1}} g_{\mu \nu}, \\
& \bar{\Phi}=\frac{n-3}{4 \alpha} \ln \Phi,
\end{aligned}
$$

where

$$
\alpha=\frac{n-3}{\sqrt{4(n-1) \omega+4 n}} .
$$

Using this conformal transformation, the action (1) transforms to

$$
\bar{I}_{G}=-\frac{1}{16 \pi} \int_{\mathcal{M}} d^{n+1} x \sqrt{-\bar{g}}\left(\bar{R}-\frac{4}{n-1}(\bar{\nabla} \bar{\Phi})^{2}-\bar{V}(\bar{\Phi})-e^{-\frac{4 \alpha \bar{\Phi}}{n-1}} \bar{F}_{\mu \nu} \bar{F}^{\mu \nu}\right)
$$

where $\bar{R}$ and $\bar{\nabla}$ are, respectively, the Ricci scalar and covariant differentiation in the spacetime metric $\bar{g}_{\mu \nu}$, and $\bar{V}(\bar{\Phi})$ is

$$
\bar{V}(\bar{\Phi})=\Phi^{-\frac{n+1}{n-1}} V(\Phi)
$$

This action is just the action of the $(n+1)$-dimensional Einstein-Maxwell-dilaton gravity, where $\bar{\Phi}$ is the dilaton field and $\bar{V}(\bar{\Phi})$ is a potential for $\bar{\Phi}$. $\quad \alpha$ is an arbitrary constant governing the strength of the coupling between the dilaton and the Maxwell field. Varying the action (7), we can obtain equations of motion

$$
\begin{aligned}
& \bar{R}_{\mu \nu}=\frac{4}{n-1}\left(\bar{\nabla}_{\mu} \bar{\Phi} \bar{\nabla}_{\nu} \bar{\Phi}+\frac{1}{4} \bar{V}(\bar{\Phi}) \bar{g}_{\mu \nu}\right)+2 e^{\frac{-4 \alpha \bar{\Phi}}{n-1}}\left(\bar{F}_{\mu \lambda} \bar{F}_{\nu}^{\lambda}-\frac{1}{2(n-1)} \bar{F}_{\rho \sigma} \bar{F}^{\rho \sigma} \bar{g}_{\mu \nu}\right) \\
& \bar{\nabla}^{2} \bar{\Phi}=\frac{n-1}{8} \frac{\partial \bar{V}}{\partial \bar{\Phi}}-\frac{\alpha}{2} e^{\frac{-4 \alpha \bar{\Phi}}{n-1}} \bar{F}_{\rho \sigma} \bar{F}^{\rho \sigma} \\
& \bar{\nabla}_{\mu}\left(e^{\frac{-4 \alpha \bar{\Phi}}{n-1}} \bar{F}^{\mu \nu}\right)=0 .
\end{aligned}
$$

Comparing Eqs. (20)-(41) with Eqs. (9)-(11), we find that if $\left(\bar{g}_{\mu \nu}, \bar{F}_{\mu \nu}, \bar{\Phi}\right)$ is the solution of Eqs. (2)-(4) with potential $\bar{V}(\bar{\Phi})$, then

$$
\left[g_{\mu \nu}, F_{\mu \nu}, \Phi\right]=\left[\exp \left(\frac{-8 \alpha \bar{\Phi}}{(n-1)(n-3)}\right) \bar{g}_{\mu \nu}, \bar{F}_{\mu \nu}, \exp \left(\frac{4 \alpha \bar{\Phi}}{n-3}\right)\right]
$$

is the solution of Eqs. (9)-(11) with potential $V(\Phi)$. 


\section{TOPOLOGICAL BLACK HOLES IN BDM THEORY}

The solutions of the field equations (99)-(11) for various metrics have been constructed by many authors (see e.g. [38, 39, 40, 41, 42, 43, 44]). Here we would like to obtain the topological black hole solutions of the field equations (2)-(4) in BDM theory, by applying the conformal transformations (12) on the corresponding solutions in the dilaton gravity theory. The $(n+1)$-dimensional topological black hole solution of the field equations (9)-(11) has been obtained by one of us in [26] for two Liouville-type dilaton potentials

$$
\bar{V}(\bar{\Phi})=2 \Lambda_{0} e^{2 \zeta_{0} \bar{\Phi}}+2 \Lambda e^{2 \zeta \bar{\Phi}}
$$

where $\Lambda_{0}, \Lambda, \zeta_{0}$ and $\zeta$ are constants. In [26] the spacetime metric was written in the form

$$
d \bar{s}^{2}=-f(r) d t^{2}+\frac{d r^{2}}{f(r)}+r^{2} R^{2}(r) h_{i j} d x^{i} d x^{j},
$$

where $f(r)$ and $R(r)$ are functions of $r$ which should be determined, and $h_{i j}$ is a function of coordinate $x_{i}$ which spanned an $(n-1)$-dimensional hypersurface with constant scalar curvature $(n-1)(n-2) k$. Here $k$ is a constant which characterized the hypersurface. Without loss of generality, one can take $k=0,1,-1$, such that the black hole horizon or cosmological horizon in (14) can be a zero (flat), positive (elliptic) or negative (hyperbolic) constant curvature hypersurface. The Maxwell equations can be integrated immediately to give

$$
\bar{F}_{t r}=\frac{q e^{\frac{4 \alpha \bar{\Phi}}{n-1}}}{(r R)^{n-1}},
$$

where $q$, an integration constant, is related to the electric charge of black hole. Defining the electric charge via $Q=\frac{1}{4 \pi} \int \exp [-4 \alpha \bar{\Phi} /(n-1)] * \bar{F} d \Omega$, we get

$$
Q=\frac{q \Omega_{n-1}}{4 \pi}
$$

where $\Omega_{n-1}$ represents the volume of constant curvature hypersurface described by $h_{i j} d x^{i} d x^{j}$. Notice that $Q$ is invariant under the conformal transformation (12). Using the ansatz

$$
R(r)=e^{\frac{2 \alpha \bar{\Phi}}{n-1}}
$$

one can show that the system of equations (9)-(10) have solutions of the form [26]

$$
f(r)=-\frac{k(n-2)\left(\alpha^{2}+1\right)^{2} b^{-2 \gamma} r^{2 \gamma}}{\left(\alpha^{2}-1\right)\left(\alpha^{2}+n-2\right)}-\frac{m}{r^{(n-1)(1-\gamma)-1}}+\frac{2 q^{2}\left(\alpha^{2}+1\right)^{2} b^{-2(n-2) \gamma}}{(n-1)\left(\alpha^{2}+n-2\right)} r^{2(n-2)(\gamma-1)}
$$




$$
\begin{gathered}
+\frac{2 \Lambda\left(\alpha^{2}+1\right)^{2} b^{2 \gamma}}{(n-1)\left(\alpha^{2}-n\right)} r^{2(1-\gamma)}, \\
R(r)=\left(\frac{b}{r}\right)^{\gamma}, \\
\bar{\Phi}=\frac{(n-1) \alpha}{2\left(1+\alpha^{2}\right)} \ln \left(\frac{b}{r}\right),
\end{gathered}
$$

where $b$ is an arbitrary constant and $\gamma=\alpha^{2} /\left(\alpha^{2}+1\right)$. In the above expression, $m$ appears as an integration constant and is related to the mass of the black hole. In order to fully satisfy the system of equations, we must have [26]

$$
\zeta_{0}=\frac{2}{\alpha(n-1)}, \quad \zeta=\frac{2 \alpha}{n-1}, \quad \Lambda_{0}=\frac{k(n-1)(n-2) \alpha^{2}}{2 b^{2}\left(\alpha^{2}-1\right)} .
$$

Notice that here $\Lambda$ is a free parameter which plays the role of the cosmological constant. Using the conformal transformation (12), the $(n+1)$-dimensional topological black hole solutions of BDM theory can be obtained as

$$
d s^{2}=-U(r) d t^{2}+\frac{d r^{2}}{V(r)}+r^{2} H^{2}(r) h_{i j} d x^{i} d x^{j}
$$

where $U(r), V(r), H(r)$ and $\Phi(r)$ are

$$
\begin{aligned}
& U(r)=-\frac{k(n-2)\left(\alpha^{2}+1\right)^{2} b^{-2 \gamma\left(\frac{n-1}{n-3}\right)} r^{2 \gamma\left(\frac{n-1}{n-3}\right)}-\frac{m b^{\left(\frac{-4 \gamma}{n-3}\right)}}{r^{n-2}} r^{\gamma\left(n-1+\frac{4}{n-3}\right)}}{\left.+\alpha^{2}-1\right)\left(\alpha^{2}+n-2\right)} \frac{2 q^{2}\left(\alpha^{2}+1\right)^{2} b^{-2 \gamma\left(n-2+\frac{2}{n-3}\right)}}{(n-1)\left(\alpha^{2}+n-2\right) r^{2\left[(n-2)(1-\gamma)-\frac{2 \gamma}{n-3}\right]}+\frac{2 \Lambda\left(\alpha^{2}+1\right)^{2} b^{2 \gamma\left(\frac{n-5}{n-3}\right)}}{(n-1)\left(\alpha^{2}-n\right)} r^{2\left(1-\frac{\gamma(n-5)}{n-3}\right)},} \\
& V(r)=-\frac{k(n-2)\left(\alpha^{2}+1\right)^{2} b^{-2 \gamma\left(\frac{n-5}{n-3}\right)} r^{2 \gamma\left(\frac{n-5}{n-3}\right)}}{\left(\alpha^{2}-1\right)\left(\alpha^{2}+n-2\right)}-\frac{m b^{\left(\frac{4 \gamma}{n-3}\right)}}{r^{n-2}} r^{\gamma\left(n-1-\frac{4}{n-3}\right)} \\
& +\frac{2 q^{2}\left(\alpha^{2}+1\right)^{2} b^{-2 \gamma\left(n-2-\frac{2}{n-3}\right)}}{(n-1)\left(\alpha^{2}+n-2\right) r^{2\left[(n-2)(1-\gamma)+\frac{2 \gamma}{n-3}\right]}}+\frac{2 \Lambda\left(\alpha^{2}+1\right)^{2} b^{2 \gamma\left(\frac{n-1}{n-3}\right)}}{(n-1)\left(\alpha^{2}-n\right)} r^{2\left(1-\gamma\left(\frac{n-1}{n-3}\right)\right)}, \\
& H(r)=\left(\frac{b}{r}\right)^{\frac{(n-5) \gamma}{n-3}}, \\
& \Phi(r)=\left(\frac{b}{r}\right)^{\frac{2(n-1) \gamma}{n-3}} .
\end{aligned}
$$

Applying the conformal transformation, the electromagnetic field and the scalar potential become

$$
\begin{aligned}
F_{t r} & =\frac{q b^{(3-n) \gamma}}{r^{(n-3)(1-\gamma)+2}}, \\
V(\Phi) & =2 \Lambda_{0} \Phi^{\frac{n\left(\alpha^{2}+1\right)+\alpha^{2}-3}{\alpha^{2}(n-1)}}+2 \Lambda \Phi^{2} .
\end{aligned}
$$


It is worth noting that in the case $k \neq 0$, these solutions are ill-defined for the string case where $\alpha=1$ (this is corresponding to $\omega=(n-9) / 4$ ). It is also notable to mention that the electric field $F_{t r}$ and the scalar field $\Phi(r)$ go to zero as $r \rightarrow \infty$. When $\omega \rightarrow \infty(\alpha=0=\gamma)$, these solutions reduce to

$$
U(r)=V(r)=k-\frac{m}{r^{n-2}}+\frac{2 q^{2}}{(n-1)(n-2) r^{2(n-2)}}-\frac{2 \Lambda}{n(n-1)} r^{2},
$$

which describes an $(n+1)$-dimensional asymptotically (anti)-de Sitter topological black holes with a positive, zero or negative constant curvature hypersurface (see e.g. [22, 23]). It is easy to show that the Kretschmann scalar $R_{\mu \nu \lambda \kappa} R^{\mu \nu \lambda \kappa}$ diverge at $r=0$ and therefore there is an essential singularity at $r=0$. As one can see from Eqs. (23)-(24), the solutions are also ill-defined for $\alpha=\sqrt{n}$ with $\Lambda \neq 0$ (corresponding to $\omega=-3(n+3) / 4 n$ ). The cases with $\alpha<\sqrt{n}$ and $\alpha>\sqrt{n}$ should be considered separately. In the first case where $\alpha<\sqrt{n}$, there exist a cosmological horizon for $\Lambda>0$, while there is no cosmological horizons if $\Lambda<0$. Indeed, in the latter case $(\alpha<\sqrt{n}$ and $\Lambda<0)$ the spacetimes exhibit a variety of possible casual structures depending on the values of the metric parameters $\alpha, m, q$ and $k$ [26]. Therefore, our solutions can represent topological black hole, with inner and outer event horizons, an extreme topological black hole, or a naked singularity provided the parameters of the solutions are chosen suitably. In the second case where $\alpha>\sqrt{n}$, the spacetime has a cosmological horizon for $\Lambda<0$ despite the value of curvature constant $k$, while for $\Lambda>0$ we have cosmological horizon in the case $k=1$ and naked singularity for $k=0,-1$.

\section{THERMODYNAMICS OF TOPOLOGICAL BD BLACK HOLE}

We now turn to the investigation of the thermodynamics of charged topological BD black holes we have just found. The Hawking temperature of the topological black hole on the outer horizon $r_{+}$can be calculated using the relation

$$
T_{+}=\frac{\kappa}{2 \pi}=\frac{U^{\prime}\left(r_{+}\right)}{4 \pi \sqrt{U / V}}
$$

where $\kappa$ is the surface gravity. Then, one can easily show that

$$
\begin{aligned}
T_{+} & =-\frac{\left(\alpha^{2}+1\right)}{2 \pi(n-1)}\left(\frac{k(n-2)(n-1) b^{-2 \gamma}}{2\left(\alpha^{2}-1\right)} r_{+}^{2 \gamma-1}+\Lambda b^{2 \gamma} r_{+}^{1-2 \gamma}+q^{2} b^{-2(n-2) \gamma} r_{+}^{(2 n-3)(\gamma-1)-\gamma}\right) \\
& =-\frac{k(n-2)\left(\alpha^{2}+1\right) b^{-2 \gamma}}{2 \pi\left(\alpha^{2}+n-2\right)} r_{+}^{2 \gamma-1}+\frac{\left(n-\alpha^{2}\right) m}{4 \pi\left(\alpha^{2}+1\right)} r_{+}{ }^{(n-1)(\gamma-1)}
\end{aligned}
$$




$$
-\frac{q^{2}\left(\alpha^{2}+1\right) b^{-2(n-2) \gamma}}{\pi\left(\alpha^{2}+n-2\right)} r_{+}{ }^{(2 n-3)(\gamma-1)-\gamma} .
$$

If we compare Eq. (31) with the temperature obtained in the dilaton gravity theory [26], we find that the temperature is invariant under the conformal transformation (12). This is due to the fact that the conformal parameter is regular at the horizon. Equation (31) also shows that when $k=0$, the temperature is negative for two cases $(i) \alpha>\sqrt{n}$ despite the sign of $\Lambda$, and $(i i)$ positive $\Lambda$ despite the value of $\alpha$. As we argued above in these two cases we encounter cosmological horizons. Physically it is not easy to accept the negative temperature, the temperature on the cosmological horizon should be defined as $T=|\kappa| / 2 \pi$ so that it becomes a positive since on the cosmological horizon the surface gravity is negative.

The ADM (Arnowitt-Deser-Misner) mass $M$, entropy $S$ and electric potential $U$ of the topological black hole can be calculated through the use of the Euclidean action method [45]. In this approach, first the electric potential and the temperature are fixed on a boundary with a fixed radius $r_{+}$. The Euclidean action has two parts; bulk and surface. The first step to make the Euclidean action is to substitute $t$ with $i \tau$. This makes the metric positive definite:

$$
d s^{2}=U(r) d \tau^{2}+\frac{1}{V(r)} d r^{2}+r^{2} H^{2}(r) h_{i j} d x^{i} d x^{j} .
$$

There is a conical singularity at the horizon $r=r_{+}$in the Euclidean metric [45]. To eliminate it, the Euclidian time $\tau$ is made periodic with period $\beta$, where $\beta$ is the inverse of Hawking temperature. Now we obtain the Euclidean action of $(n+1)$-dimensional BransDicke-Maxwell theory. The Euclidean action can be calculated analytically and continuously changing of action (1) to Euclidean time $\tau$, i.e.,

$$
I_{G E}=-\frac{1}{16 \pi} \int_{\mathcal{M}} d^{n+1} x \sqrt{g}\left(\Phi R-\frac{\omega}{\Phi}(\nabla \Phi)^{2}-V(\Phi)-F_{\mu \nu} F^{\mu \nu}\right)-\frac{1}{8 \pi} \int d^{n} x \sqrt{h} \Phi\left(K-K_{0}\right),
$$

where $K_{0}$ is the trace of the extrinsic curvature on the metric $h$ for our metric background with $q=0$ and $m=0$, which must be added so that it can normalize the Euclidean action to zero in this spacetime [46]. Using the metric (32), we find

$$
\begin{aligned}
& R=-g^{-1 / 2}\left(g^{1 / 2} U^{\prime} V / U\right)^{\prime}-2 G_{0}^{0}, \\
& K=-\frac{\sqrt{V}\left[r H U^{\prime}+2(n-1)\left(U H+r U H^{\prime}\right)\right]}{2 r H U},
\end{aligned}
$$

where $G_{0}^{0}$ is the (00) component of the Einstein tensor. Inserting $U(r)$ and $V(r)$ from (23) and (24) with $q=0$ and $m=0$ in $K$ we obtain the extrinsic curvature for the metric 
background

$$
\begin{aligned}
K_{0}= & \left(\frac{b}{r}\right)^{\frac{2 \gamma}{n-3}}\left(-\frac{k(n-2)\left(\alpha^{2}+1\right)^{2} b^{-2 \gamma} r^{2 \gamma}}{\left(\alpha^{2}-1\right)\left(\alpha^{2}+n-2\right)}+\frac{2 \Lambda\left(\alpha^{2}+1\right)^{2} b^{2 \gamma} r^{2-2 \gamma}}{(n-1)\left(\alpha^{2}-n\right)}\right)^{1 / 2} \\
& \times\left[2 b^{2 \gamma}\left(\alpha^{2}-1\right)(n \gamma-n+3-5 \gamma)\left(\alpha^{2}+n-2\right) n \Lambda r^{2-2 \gamma}\right. \\
& \left.+(n-2) r^{2 \gamma} b^{-2 \gamma}\left(n-\alpha^{2}\right)(n-1)^{2} k(\gamma n-n-6 \gamma+3)\right](n-3)^{-1} r^{-1} \\
& \times\left[2 b^{2 \gamma} \Lambda\left(\alpha^{2}-1\right)\left(\alpha^{2}+n-2\right) r^{2-2 \gamma}+r^{2 \gamma} b^{-2 \gamma} k(n-1)(n-2)\left(n-\alpha^{2}\right)\right]^{-1}
\end{aligned}
$$

Substituting Eqs. (34)-(36) in action (33) and using Eqs. (23)-(28), after a long calculation, we obtain the Euclidean action as

$$
I_{G E}=\beta \frac{\Omega_{n-1}}{16 \pi}\left(\frac{b^{(n-1) \gamma}(n-1) m}{\left(\alpha^{2}+1\right)}\right)-\frac{\Omega_{n-1}}{4}\left(b^{(n-1) \gamma} r_{+}^{(n-1)(1-\gamma)}\right)-\beta \frac{\Omega_{n-1} q^{2}}{8 \pi \Upsilon r_{+}^{\Upsilon}},
$$

where $\Upsilon=(n-3)(1-\gamma)+1$. According to Ref. [46, 47, 48, 49], the thermodynamical potential can be given by $I_{G E}$, we get

$$
I_{G E}=\beta M-S-\beta U q,
$$

where $M$ is the ADM mass, $S$ and $U$ are, respectively, the entropy and the electric potential. Comparing Eq. (37) with Eq. (38), we find

$$
\begin{gathered}
M=\frac{b^{(n-1) \gamma}(n-1) \Omega_{n-1}}{16 \pi\left(\alpha^{2}+1\right)} m, \\
S=\frac{b^{(n-1) \gamma} r_{+}^{(n-1)(1-\gamma)}}{4} \Omega_{n-1}, \\
U=\frac{q b^{(3-n) \gamma} \Omega_{n-1}}{\Upsilon r_{+}^{\Upsilon}} .
\end{gathered}
$$

Comparing the conserved and thermodynamic quantities calculated in this section with those obtained in [26], we find that they are invariant under the conformal transformation (12). It is worth emphasizing that in BD theory, where we have the additional gravitational scalar degree of freedom, the entropy of the black hole does not follow the area law [50]. This is due to the fact that the black hole entropy comes from the boundary term in the Euclidean action formalism. Nevertheless, the entropy remains unchanged under the conformal transformations. Finally, we consider the first law of thermodynamics for the topological black hole. It is a matter of calculation to show that the the conserved and thermodynamic quantities obtained above satisfy the first law of black hole thermodynamics

$$
d M=T d S+U d Q
$$




\section{SUMMARY AND DISCUSSION}

To conclude, in $(n+1)$-dimensions, when the $(n-1)$-sphere of black hole event horizn is replaced by an $(n-1)$-dimensional hypersurface with positive, zero or negative constant curvature, the black hole is called as a topological black hole. The construction and analysis of these exotic black holes in anti-de Sitter (AdS) space is a subject of much recent interest. This interest is motivated by the correspondence between the gravitating fields in an AdS spacetime and conformal field theory on the boundary of the AdS spacetime. In this paper, we further generalized these exotic solutions by constructing a class of $(n+1)$-dimensional $(n \geq 4)$ topological black holes in BDM theory in the presence of a potential for the scalar field. In contrast to the topological black holes in the Einstein-Maxwell theory, which are asymptotically AdS, the topological black holes we found here, are neither asymptotically flat nor (A)dS. Indeed, the scalar potential plays a crucial role in the existence of these black holes, as the negative cosmological constant does in the Einstein-Maxwell theory. When $k= \pm 1$, these solutions do not exist for the string case where $\alpha=1$ (corresponding

to $\omega=(n-9) / 4$ ). Besides they are ill-defined for $\alpha=\sqrt{n}$ with $\Lambda \neq 0$ (corresponding to $\omega=-3(n+3) / 4 n)$. We obtained the conserved and thermodynamic quantities through the use of the Euclidean action method, and verified that the conserved and thermodynamic quantities of the solutions satisfy the first law of black hole thermodynamics. We found that the entropy does not satisfy the area law. We also found that the conserved and thermodynamic quantities are invariant under the conformal transformation.

\section{Acknowledgments}

This work has been supported financially by Research Institute for Astronomy and Astrophysics of Maragha, Iran.

[1] C. Brans and R. H. Dicke, Phys. Rev. 124, 925 (1961).

[2] C. M. Will, Theory and Experiment in Gravitational Physics, (Cambridge University Press, Cambridge, 1993).

[3] A. Sen, Phys. Rev. Lett. 69, 1006 (1992). 
[4] G. W. Gibbons and K. Maeda, Ann. Phys. (N.Y.) 167, 201 (1986).

[5] V. Frolov, A. Zelinkov and U. Bleyer, Ann. Phys. (Leipzig) 44, 371 (1987).

[6] C. H. Brans, Phys. Rev. 125, 2194 (1962).

[7] S. W. Hawking, Commun. Math. Phys. 25, 167 (1972).

[8] R. G. Cai and Y. S. Myung, Phys. Rev. D. 56, 3466 (1997).

[9] H. Kim, Phys. Rev. D, 60, 024001 (1999).

[10] M. H. Dehghani, J. Pakravan, and S. H. Hendi, Phys. Rev. D 74, 104014 (2006).

[11] C. J. Gao and S. N. Zhang, gr-qc/0604083.

[12] H. Kim, Nuovo Cim. B 112 (1997) 329.

[13] S. W. Hawking and G. F. Ellis. The large scale structure of spacetime (Cambridge University Press, Cambridge, England, 1973).

[14] S. W. Hawking. Commun. Math. Phys. 25, 152 (1972).

[15] J. L. Friedman, K. Schleich and D. M. Witt, Phys. Rev. Lett. 71, 1486 (1993).

[16] J. L. Friedman, K. Schleich, and D. M. Witt, Phys. Rev. Lett. 75, 1872 (1995).

[17] S. W. Hawking and D. N. Page, Commun. Math. Phys. 87, 577 (1983).

[18] D. Birmingham, Class. Quant. Gravit. 16, 1197 (1999).

[19] J. P. S. Lemos, Phys. Lett. B 353, 46 (1995).

[20] R. G. Cai and Y. Z. Zhang, Phys. Rev. D 54, 4891 (1996).

[21] L. Vanzo, Phys. Rev. D 56, 6475 (1997).

[22] D. R. Brill, J. Louko, and P. Peldan, Phys. Rev. D 56, 3600 (1997).

[23] R. G. Cai and K. S. Soh, Phys. Rev. D 59 (1999) 044013.

[24] R. G. Cai, J.Y. Ji, and K. S. Soh, Phys. Rev. D 57(1998) 6547.

[25] A. Sheykhi, Phys. Letts. B 662 (2008) 7.

[26] A. Sheykhi, Phys. Rev. D 76(2007) 124025 .

[27] J. Crisostomo, R. Troncoso, and J. Zanelli, Phys. Rev. D 62, 084013 (2000).

[28] R. Aros, R. Troncoso, and J. Zanelli, Phys. Rev. D 63, 084015 (2001).

[29] R. G. Cai, Phys. Rev. D 65, 084014 (2002).

[30] M. H. Dehghani, Phys. Rev. D 70, 064019 (2004).

[31] J. P. Lemos, Class. Quant. Gravit. 12 (1995) 1081.

[32] C. G. Huang and C. B. Liang, Phys. Lett. A 201(1995) 27.

[33] R. G. Cai, Nucl. Phys. B 524 (1998)639. 
[34] R. B. Mann, Class. Quant. Gravit. 14, L109 (1997).

[35] M. Banados, A. Gomberoff, and C. Martnez, Class. Quant. Gravit. 15 (1998) 3575.

[36] R. G. Cai, Phys. Rev. D 65(2002) 084014.

[37] R. G. Cai, Phys. Lett. B 582(2004) 237.

[38] K. C. K. Chan, J. H. Horne and R. B. Mann, Nucl. Phys. B447 (1995) 441.

[39] R. G. Cai and Y. Z. Zhang, Phys. Rev D 64 (2001) 104015.

[40] G. Clement, D. Gal'tsov and C. Leygnac, Phys. Rev. D 67(2003) 024012.

[41] M. H Dehghani and N. Farhangkhah, Phys. Rev. D 71 (2005) 044008.

[42] M. H Dehghani, Phys. Rev. D 71 (2005) 064010.

[43] A. Sheykhi, M. H. Dehghani, N. Riazi, Phys. Rev. D 75 (2007) 044020.

[44] A. Sheykhi, M. H. Dehghani, N. Riazi and J. Pakravan Phys. Rev. D 74 (2006) 084016.

[45] R. G. Cai, R. K. Su, and P. K. N. Yu, Phys. Lett. A 195, (1994) 307.

[46] J.D. Brown, E.A. Martinez and J.W. York Jr., Phys. Rev. Lett. 66 (1991) 2281.

[47] H.W. Braden, J.D. Brown, B.F. Whiting and J.W. York Jr., Phys. Rev. D 42 (1990) 3376.

[48] B.F. Whiting and J.W. York Jr., Phys. Rev. Lett. 61 ( 1988 ) 1336.

[49] J.W. York Jr., Phys. Rev. D 33 ( 1986 ) 2092.

[50] G. Kang, Phys. Rev. D 54, (1996) 7483. 